\title{
Slowing down of spin glass correlation length growth: Simulations meet experiments
}

\author{
Qiang Zhai, ${ }^{1}$ V. Martin-Mayor $\odot,{ }^{2,3}$ Deborah L. Schlagel, ${ }^{4}$ Gregory G. Kenning, ${ }^{5}$ and Raymond L. Orbach ${ }^{1}$ \\ ${ }^{1}$ Texas Materials Institute, The University of Texas at Austin, Austin, Texas 78712, USA \\ ${ }^{2}$ Departamento de Física Teórica, Universidad Complutense, 28040 Madrid, Spain \\ ${ }^{3}$ Instituto de Biocomputación y Física de Sistemas Complejos, 50018 Zaragoza, Spain \\ ${ }^{4}$ Division of Materials Science and Engineering, Ames Laboratory, Ames, Iowa 50011, USA \\ ${ }^{5}$ Department of Physics, Indiana University of Pennsylvania, Indiana, Pennsylvania 15705, USA
}

(Received 5 May 2019; revised manuscript received 5 September 2019; published 24 September 2019)

\begin{abstract}
The growth of the spin glass correlation length has been measured as a function of the waiting time $t_{\mathrm{w}}$ on a single crystal of CuMn (6 at. \%), reaching values $\xi \sim 150 \mathrm{~nm}$, larger than any other glassy correlation length measured to date. We find an aging rate $d \ln t_{\mathrm{w}} / d \ln \xi$ larger than found in previous measurements, which evinces a dynamic slowing down as $\xi$ grows. Our measured aging rate is compared with simulation results by the Janus Collaboration. After critical effects are taken into account, we find excellent agreement with the Janus data.
\end{abstract}

DOI: 10.1103/PhysRevB.100.094202

\section{INTRODUCTION}

The accuracy provided by superconducting quantum interference devices in measurements of the response to an externally applied magnetic field puts spin glasses in a privileged status among glassy systems [1] in at least two respects. First, we know that their sluggish dynamics originates in a bona fide phase transition at a critical temperature $T_{\mathrm{c}}$, separating the paramagnetic phase from the low-temperature glassy phase [2]. Second, the suspected mechanism for the dynamic slowdown, namely, the increasing size of the cooperative regions [3], has been confirmed experimentally [4]. The size of these cooperative regions, the so-called spin glass correlation length $\xi$, was found to be as large as $\xi \approx 80 \mathrm{~nm}$ (much larger than found to date in other glassy systems, glycerol, for instance [5]).

In the typical setup, the spin glass is rapidly cooled from high temperatures to a working temperature $T<T_{\mathrm{c}}$, where it relaxes for a waiting time $t_{\mathrm{w}}$. In principle, the growth of the correlation length $\xi\left(t_{\mathrm{w}}\right)$ is unbounded in the spin glass phase (however, finite crystallite sizes play a role; see below). Much attention has been paid to the (renormalized) aging rate

$$
z_{\mathrm{c}}(T, \xi)=\frac{T}{T_{\mathrm{c}}} \frac{d \ln t_{\mathrm{w}}}{d \ln \xi}
$$

The renormalizing factor $T / T_{\mathrm{c}}$ makes $z_{\mathrm{c}}(T, \xi) \approx z_{\mathrm{c}}(\xi)$ [6]. Hence, Eq. (1) can be rephrased as $t_{w}^{\text {eff }} \approx \tau_{0} \exp \{[\Delta(\xi)-$ $\left.\left.E_{z}(H)\right] / k_{B} T\right\}$, where $\tau_{0}=\hbar /\left(k_{\mathrm{B}} T_{\mathrm{c}}\right)$ is the exchange time, $E_{z}$ is the Zeeman energy, and $\Delta(\xi)$ is a free-energy barrier.

In fact, values of $z_{\mathrm{c}}$ have been found to vary from system to system. For a bulk, polycrystalline sample of CuMn (6 at. \%), Joh et al. [4] found at a reduced temperature $T / T_{\mathrm{c}}=0.89$, $z_{\mathrm{c}}=5.917$. For a polycrystalline bulk thiospinel, Joh et al. found at a reduced temperature of $T / T_{\mathrm{c}}=0.72, z_{\mathrm{c}}=7.576$. There is no way of knowing the crystallite size in these "bulk" measurements, but they were certainly larger than the thin-film thicknesses of Zhai et al. [7]. Zhai et al. found, for CuMn (11.7 at. \%), thin films at reduced temperatures of
$T / T_{\mathrm{c}}=0.43,0.59,0.78, \quad z_{\mathrm{c}}=9.62$. Working at $T / T_{\mathrm{c}}=$ 0.95 , Kenning et al. [8] obtained $z_{\mathrm{c}}=6.80$ in a bulk polycrystalline CuMn (5 at. \%) sample.

Some hints to classify these apparently conflicting results can be found in a recent large-scale numerical simulation by the Janus Collaboration [9] (using the custom-built computer Janus II [10]). They computed $\xi$ in the time range $10^{-12} \mathrm{~s}$ $\leqslant t_{\mathrm{w}} \leqslant 0.1 \mathrm{~s}$ for temperatures $0.5 \leqslant T / T_{\mathrm{c}} \leqslant 1$. In fact, $\xi$ varied by a larger factor in the simulation than in experiments: close to $T_{\mathrm{c}}$, from $\xi \sim a_{0}$ to $\xi \sim 17 a_{0}\left(a_{0}\right.$ is the typical distance between magnetic moments). Yet the maximum $\xi / a_{0}$ reached in the simulations was smaller than experiment by a factor of approximately 10 .

The Janus simulation evinced different behaviors at $T_{\mathrm{c}}$ and at $T<T_{\mathrm{c}}$ [9], according to the value of the crossover variable:

$$
x\left(t_{\mathrm{w}}, T\right)=\ell_{\mathrm{J}}(T) / \xi\left(t_{\mathrm{w}}, T\right),
$$

where $\ell_{\mathrm{J}}(T)$ is the Josephson length [11]. For $x \ll 1$ we have $T<T_{\mathrm{c}}$ behavior, while for $x \gg 1$ we find critical scaling. Because $\ell_{\mathrm{J}}(T)$ diverges at $T_{\mathrm{c}}$ as $\ell_{\mathrm{J}}(T) \propto 1 /\left(T_{\mathrm{c}}-T\right)^{\nu}$, $v=2.56(4)$ [12], $\xi\left(t_{\mathrm{w}}\right)$ needed to demonstrate lowtemperature behavior, i.e., $x \ll 1$, grows enormously upon approaching $T_{\mathrm{c}}$. For $x \ll 1, z_{\mathrm{c}}$ grows with $\xi$, but it is $T$ independent [9]. Furthermore, a mild extrapolation from $z_{\mathrm{c}}\left(\xi=12 a_{0}\right)$ to $z_{\mathrm{c}}\left(\xi=38 a_{0}\right)$ [9] is compatible with the thin-film value $z_{\mathrm{c}}=9.62$ [7] (the film width was $\sim 38 a_{0}$ ). For $x \gg 1$, the $\xi$-independent $z_{\mathfrak{c}}\left(T=T_{\mathrm{c}}\right)=6.69 \pm 0.06$ [9] agrees with the CuMn result at $T=0.95 T_{\mathrm{c}}, z_{\mathrm{c}}=6.80$ [8].

However, in spite of the just quoted agreement between experimental results and the Janus simulations, the reader might worry because CuMn is a Heisenberg spin glass, while the Janus Collaboration simulates the Ising-Edwards-Anderson model. In fact, there is theoretical ground for the success of the Ising spin glass simulations: small anisotropies such as Dzyaloshinskii-Moriya interactions [13] are present in any spin glass sample. These interactions, although tiny, extend over dozens of lattice spacings, which magnifies their effect. In fact, we know that Ising is the ruling universality class 
in the presence of coupling anisotropies [14] (the effect of anisotropies, even if negligible at small $\xi$, is strongly enhanced when $\xi$ grows [15]), which probably explains why high-quality measurements on GeMn are excellently fit with Ising scaling laws [16].

Here, we report measurements of $\xi\left(t_{\mathrm{w}}\right)$ on a single crystal of CuMn (6 at. \%) at $T=0.886 T_{\mathrm{c}}$ and for times $2 \times 10^{3} \mathrm{~s}$ $\leqslant t_{\mathrm{w}} \leqslant 8 \times 10^{4} \mathrm{~s}$. In the absence of crystallites limiting $\xi$ to the crystallite size $(\sim 80 \mathrm{~nm}$, typically), we reach $\xi \sim 150 \mathrm{~nm}$, the largest reported for a glassy phase (and, certainly, in the low-temperature regime $x \ll 1)$. Our measured aging rate $z_{c}=12.37 \pm 1.07$ is the largest reported for a spin glass, in a dramatic demonstration of the dynamic slowing down with growth of $\xi$ [9]. We are also able to reproduce our experimental results by means of a simple extrapolation of the Janus simulations [9].

The layout of the remainder of this paper is as follows. In Sec. II we provide details of our single-crystal sample. Our experimental protocol is explained in Sec. III. Our extrapolation from the Janus simulations is compared with the experimental results in Sec. IV. We present our conclusions in Sec. V. The paper ends with a number of Appendixes where more technical details are given.

\section{SAMPLE PREPARATION}

The $\mathrm{Cu}_{94} \mathrm{Mn}_{6}$ sample was prepared using the Bridgman method. The $\mathrm{Cu}$ and $\mathrm{Mn}$ were arc melted several times in an argon environment and cast in a copper mold. The ingot was then processed in a Bridgman furnace. Both x-ray fluorescence and optical observation showed that the beginning of the growth is a single phase. More details can be found in Appendix A.

\section{EXPERIMENTAL PROTOCOL}

We follow the method introduced by Joh et al. [4] for the extraction of $\xi\left(t_{\mathrm{w}}\right)$, standard in experimental work (see, e.g., $[17,18])$ and studied theoretically [19].

Specifically, the CuMn sample was quenched from 70 to $28 \mathrm{~K}$ in zero magnetic field $\left(T_{g}=31.5 \mathrm{~K}\right.$ as determined from the temperature at which the remanence disappeared). This measurement temperature was determined by two factors. Measuring at a higher temperature would have increased the Josephson length, increasing $x\left(t_{w}, T\right)$ according to Eq. (2). It was important to keep $x\left(t_{w}, T\right)$ as small as possible in order to have $T<T_{c}$ behavior. In addition, the signal-to-noise ratio diminishes as the measuring temperature $T$ increases. The lower $T$ is, the slower is the dynamics. The working temperature $T=28 \mathrm{~K}$ was chosen to keep the measurements within laboratory timescales.

The system was aged for a time $t_{w}$ after the temperature was stabilized; then a magnetic field $H$ was applied, and $24 \mathrm{~s}$ after the field stabilized, the zero-field magnetization $M_{\mathrm{ZFC}}(t, T)$ was recorded ( $t$ is the time that has elapsed since the magnetic field was switched on). In this set of experiments, $t_{w}$ was set as 2000, 2750, 3420, 5848, $10000,20000,40000$, and $80000 \mathrm{~s}$, with magnetic fields of $20,32,47$, and 59 Oe. The latter are used for the magnetic field dependence of the effective waiting time $t_{w}^{\text {eff }}$ as determined from the time for the relaxation function to reach its maximum as a function of $\ln t$,

$$
S(t)=\frac{d M_{\mathrm{ZFC}}(t)}{d \ln t} .
$$

Note that the effective waiting time $t_{w}^{\text {eff }}$ where $S(t)$ attains its maximum depends on the applied magnetic field because the Zeeman effect lowers the free-energy barrier heights. This results in a shift of the peak in $S(t)$ (its maximum $t_{w}^{\text {eff }}$ ):

$$
\Delta_{\max }-N_{c} \chi H^{2}=k_{B} T \ln t_{w}^{\text {eff }}-k_{B} T \ln \tau_{0},
$$

where $N_{c}$ is the number of spin glass correlated spins, $\chi$ is the spin glass field-cooled susceptibility per spin $\left[M_{\mathrm{FC}} /(N H)\right.$, with $N$ being the total number of Mn spins in the sample], and $\tau_{0}$ is an effective exchange time $\tau_{0} \sim \hbar /\left(k_{\mathrm{B}} T_{g}\right)$. The beauty of this expression is that $N_{c}$ can be determined from Eq. (3) from the measurement of the peak position of $S(t)$ as a function of $H^{2}$ and from other known values of the parameters. A representative set of data is exhibited in Fig. 1. Our $t_{w}^{\text {eff }}$ are listed in Table I.

Knowing $N_{c}$, the correlation length $\xi$ can be generated from the relationship [20]

$$
N_{c} \approx\left(\frac{\xi}{a_{0}}\right)^{d_{\mathrm{f}}}
$$
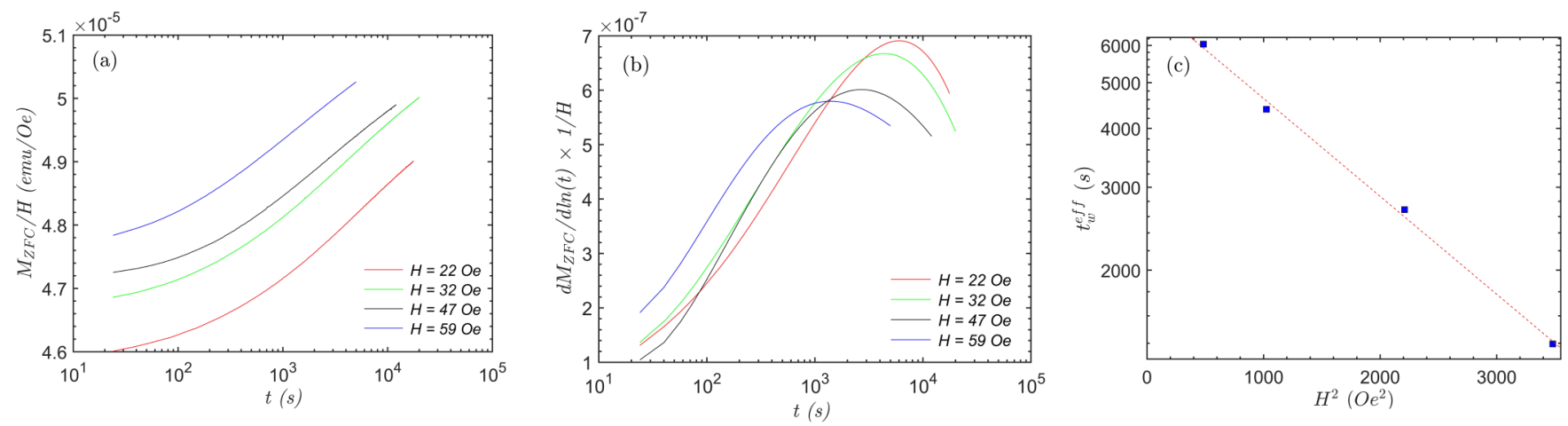

FIG. 1. A representative set of data. The three figures are for a waiting time $t_{w}=10 \mathrm{ks}$. (a) A plot of the measured zero-field susceptibility $M_{Z F C} / H$ as a function of time. (b) The response function, $S(t)=d\left(M_{Z F C} / H\right) / d(\ell n t)$, as a function of time for varying values of the applied magnetic field $H$, the peak of which defines $t_{w}^{\text {eff }}$. (c) A plot of $\ell n t_{w}^{\text {eff }}$ vs $H^{2}$. 
TABLE I. Effective waiting time $t_{w}^{\text {eff }}$ extracted from the zerofield-cooled magnetization aging experiments.

\begin{tabular}{lcccc}
\hline \hline$t_{w}(\mathrm{~s})$ & $H=22 \mathrm{Oe}$ & $H=32 \mathrm{Oe}$ & $H=47 \mathrm{Oe}$ & $H=59 \mathrm{Oe}$ \\
\hline 2000 & 1463 & 1161 & $727^{\mathrm{a}}$ & 593 \\
2750 & 1924 & 1599 & 1009 & 696 \\
3420 & 2395 & 1832 & 1069 & 726 \\
5848 & 3860 & 2865 & 1615 & 1058 \\
10000 & 6038 & 4390 & 2689 & 1395 \\
20000 & 11978 & 8073 & 4047 & 2104 \\
40000 & 21710 & 14601 & 6838 & 3451 \\
80000 & 41748 & 26215 & 11467 & 5266 \\
\hline \hline
\end{tabular}

${ }^{\text {a }}$ Measured in 50 Oe.

where $d_{\mathrm{f}}$ is the fractal dimension equal to $d_{\mathrm{f}}=d-\theta / 2$ $(d=3$ is the space dimension, while $\theta$ is the so-called replicon exponent [19]). Because at the correlation lengths of interest $\theta \approx 0.3$, the approximation $d_{\mathrm{f}} \approx d$ made in previous work (Ref. [4], for instance) does not introduce a significant error. In fact, the exponent $\theta$ has a small dependence on $\xi$ [21]. We have solved this problem by taking the exponent $\theta(\xi)$ from Ref. [9] and then solved for $\xi$ in Eq. (5) self-consistently (see Appendix C). The appropriate value of $\theta$ turns out to be $\theta \approx 0.34$. The outcome of this analysis is shown in Fig. 2. The estimated Josephson length at our working temperature is $\ell_{\mathrm{J}}=21.82 a_{0}\left(a_{0}=0.64 \mathrm{~nm}\right.$ in our sample; see Ref. [9] and Appendix B). Hence, the crossover variable in our experiment is in the range $0.091 \leqslant x \leqslant 0.12$, so that we can be reasonably sure to be free from critical effects. The resulting aging rate is $z_{c}=12.37 \pm 1.07$. Compared with previous values of $z_{c}$, obtained in experiments reaching a smaller $\xi\left(t_{w}, T\right)[4,7,8]$, this is the largest aging rate reported for a spin glass, which shows that the growth of $\xi$ is indeed slowing down with increasing $\xi$.

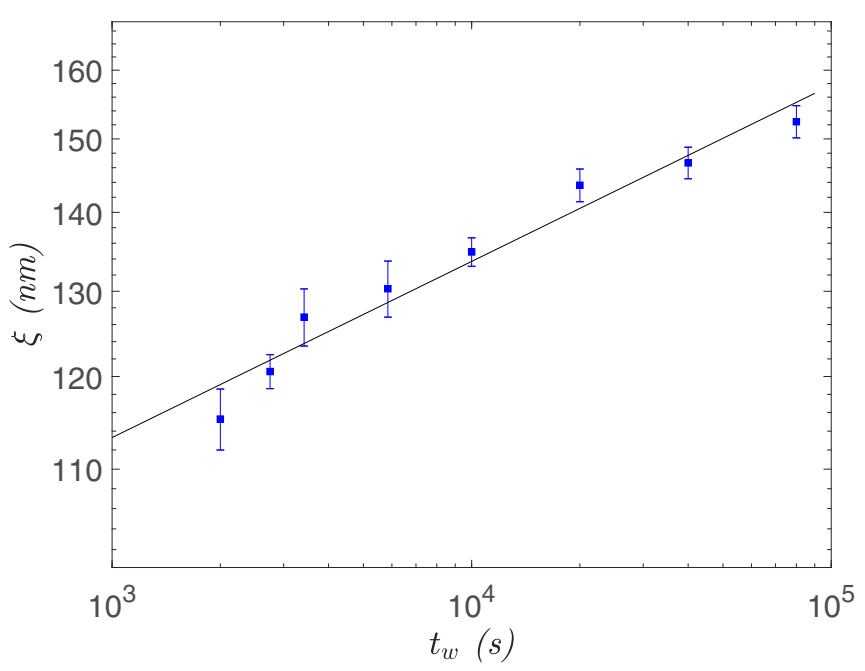

FIG. 2. $\xi\left(t_{w}, T\right)$ as a function of waiting time $t_{w}$ at a measuring temperature $T=28 \mathrm{~K}$ (the transition temperature is $T_{\mathrm{c}} \approx 31.5 \mathrm{~K}$ ). The straight line is a fit to $\ln t_{\mathrm{w}}=\left(z_{\mathrm{c}} T_{\mathrm{c}} / T\right) \ln \xi+$ const [recall Eq. (1)], yielding $z_{c}=12.37 \pm 1.07$.

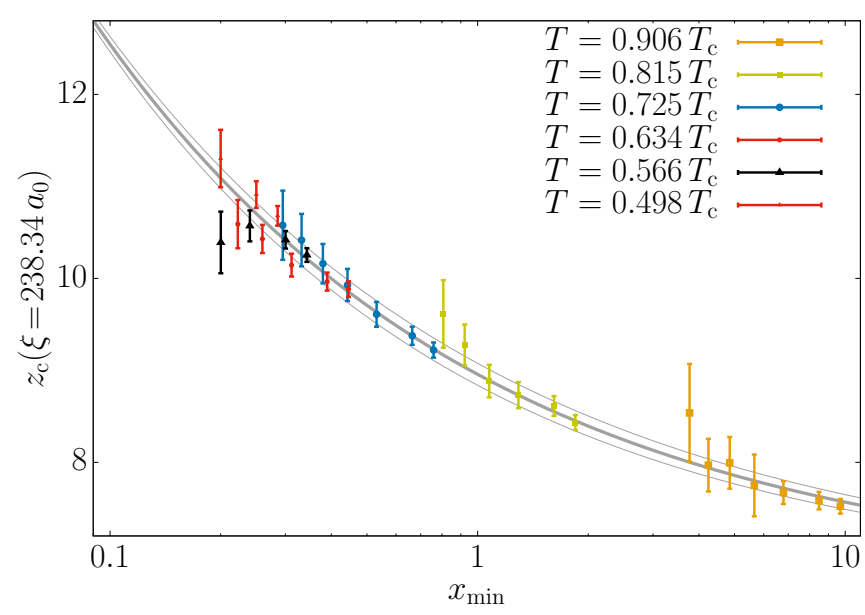

FIG. 3. The estimates from different temperatures and minimal correlation lengths for the aging rate at $\xi_{\text {target }}=238.34 a_{0}$ (our largest) are a simple function of the crossover variable $x_{\min }=$ $\ell_{\mathrm{J}}(T) / \xi_{\min }$ [see Eq. (7)]. The central gray line is a fit to Eq. (7) with figure of merit $\chi^{2} / \mathrm{DOF}=24.5 / 30$ [DOF $=$ degrees of freedom; the fit generates the exponent $\beta\left(\xi_{\text {target }}=238.34 a_{0}\right)=0.41$, and the dependency on $\xi_{\text {target }}$ of exponent $\beta$ turns out to be small]. The upper (lower) gray line is a fit to the data plus (minus) the error bar. The estimates of $z_{\mathrm{c}}$ for the different $\left(T, \xi_{\min }\right)$ were obtained by applying Eq. (6) to the data in Table III of the Supplemental Material of Ref. [9] (see Appendix D for details).

\section{EXTRAPOLATIONS FROM SIMULATIONS}

The main problem to overcome is the crossover between critical scaling and the $T<T_{\mathrm{c}}$ physics. Indeed, the largest correlation length reached in the simulations is $\xi=17.3 a_{0}$ at $T=0.905 T_{\mathrm{c}}$ [9], which results in a very large crossover variable $x=1.96$. Much smaller values of $x$ were reached in the simulations but at lower $T$ [9]. Therefore, we need to consider the full data set for $T<T_{\mathrm{c}}$ in Table III of the Supplemental Material of Ref. [9]. We shall only outline our analysis here and refer the reader to Appendix D for full details. To ease comparison with [9], we give $\xi$ in units of $a_{0}$ from now on (recall that $a_{0}=0.64 \mathrm{~nm}$ for our sample).

We should mention that two possibilities were considered in Ref. [9] for extrapolating the simulation's $z_{\mathrm{c}}$ to larger values of $\xi$. One was Saclay's ansatz for the crossover to activated dynamics [22,23], which, however, yields too high a $z_{\mathrm{c}}$ [9] when applied to the thin-film experiments [7]. Therefore, we focus on the convergent ansatz for extrapolating $z_{\mathrm{c}}$ to correlation length $\xi_{\text {target }}$ by taking into account only data with $\xi \geqslant \xi_{\min }(\hat{\omega}=0.35)[9]$,

$$
z_{\mathrm{c}}\left(T, \xi_{\text {target }}, \xi_{\text {min }}\right)=\frac{T}{T_{\mathrm{c}}}\left[z_{\infty}\left(T, \xi_{\text {min }}\right)+\frac{A\left(T, \xi_{\text {min }}\right)}{\xi_{\text {target }}^{\hat{\omega}}}\right] .
$$

Now, when applying Eq.(6) to any $\xi_{\text {target }}$, we end up with as many predicted aging rates as pairs of $\left(T, \xi_{\min }\right)$ were considered in the simulations. Fortunately, these many predictions (see Fig. 3) can be nicely organized as a function of the crossover variable $x_{\min }=\ell_{\mathrm{J}}(T) / \xi_{\min }$ [24]:

$$
z_{\mathrm{c}}\left(T, \xi_{\text {target }}, \xi_{\min }\right)=6.69+\frac{\alpha\left(\xi_{\text {target }}\right)}{x_{\text {min }}^{\beta\left(\xi_{\text {target }}\right)}} .
$$




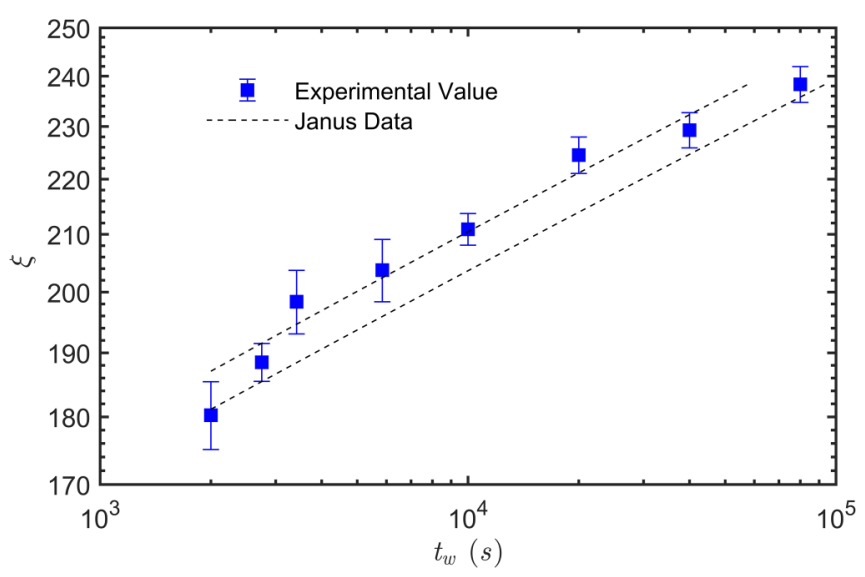

FIG. 4. The experimental correlation length from Fig. 2, as measured in units of the average distance between magnetic moments $a_{0}=0.64 \mathrm{~nm}$, is shown as a function of the waiting time. The two lines were obtained from our extrapolations from the simulations by the Janus Collaboration [9] [recall Eqs. (8) and (10)]. The two lines are the two extremal curves compatible with the initial condition taken from our experiment, $\xi^{*}\left(t_{\mathrm{w}}^{*}=2750 \mathrm{~s}\right)=(188.5 \pm 3) a_{0}$.

Thus, our final extrapolation at $T=28 \mathrm{~K}$ is

$$
z_{\mathrm{c}}\left(\xi_{\text {target }}\right)=6.69+\frac{\alpha\left(\xi_{\text {target }}\right)}{x_{\text {target }}^{\beta\left(\xi_{\text {target }}\right)}}, \quad x_{\text {target }}=\frac{\ell_{\mathrm{J}}(28 \mathrm{~K})}{\xi_{\text {target }}},
$$

$\left[\alpha\left(\xi_{\text {target }}\right)\right.$ and $\beta\left(\xi_{\text {target }}\right)$ come from the fit to Eq. (7); recall Fig. 3]. We obtain in this way

$$
z_{\mathrm{c}}\left(180.26 a_{0}\right)=11.94 \pm 0.08, z_{\mathrm{c}}\left(238.34 a_{0}\right)=12.76 \pm 0.08
$$

Both extrapolations are in excellent agreement with the experimental result $z_{c}=12.37 \pm 1.07$ from Fig. 2 (roughly speaking, $z_{c}=12.37 \pm 1.07$ is an average of $z_{c}(\xi)$ in the range $\left.180.26 a_{0} \leqslant \xi \leqslant 238.34 a_{0}\right)$. We stress that the extrapolations (9) took no input from the experiment other than the values of $\xi_{\text {target }}$. However, by recalling [see Eq. (1)]

$$
\ln t_{\mathrm{w}}-\ln t_{\mathrm{w}}^{*}=\int_{\ln \xi^{*}}^{\ln \xi} d\left(\ln \xi^{\prime}\right) \frac{T_{\mathrm{c}}}{T} z_{\mathrm{c}}\left(\xi^{\prime}\right)
$$

and borrowing the initial condition $\xi^{*}\left(t_{\mathrm{w}}^{*}=2750 \mathrm{~s}\right)$ from the experiment, we obtain a fairly satisfactory comparison between our experiment and our extrapolations from the Janus simulations in Fig. 4. We note as well that the initial condition $\xi^{*}\left(t_{\mathrm{w}}=2000 \mathrm{~s}\right)$ from the experiment, afflicted by larger errors and short-time systematic effects, produces similar extrapolated curves.

\section{CONCLUSIONS}

We have reported an experimental measurement of the spin glass correlation length in a single-crystal sample of CuMn (6 at. \%). Our experiment is free from two systematic effects encountered in previous work: (i) the growth of the correlation length is not hampered by the sample geometry (neither crystallites [4] nor the film thickness [7]), and (ii) our results are representative of the low-temperature phase (i.e., they are not contaminated by critical scaling), as shown by the small value of the crossover variable we reach [recall Eq. (2)].
We reported the largest spin-glass correlation length reported for a glassy phase. Our aging rate is also the largest reported to date (at least as measured in a spin glass). We thus confirm the slowing down as $\xi$ grows that was suggested by the simulations of the Janus Collaboration [9]. Furthermore, we have been able to reproduce our experimental results by means of a simple extrapolation of the Janus results. We believe this relation between simulations and experiment opens new opportunities in condensed-matter physics. The complementary contributions allow exploration of phenomena, especially in complex systems, with the particular insights of each partner fueling the interpretation and development of the other. This paper is the beginning of this new research relationship.

\section{ACKNOWLEDGMENTS}

We warmly thank the Janus Collaboration for allowing us to reanalyze their results. We also thank L. A. Fernández for his help with figure preparation. We are grateful for helpful discussions with S. Swinnea about sample characterization. This work was partially supported by the U.S. Department of Energy, Office of Basic Energy Sciences, Division of Materials Science and Engineering, under Award No. DESC0013599 and Contract No. DE-AC02-07CH11358, and by the Ministerio de Economía, Industria y Competitividad (MINECO; Spain) through Grants No. FIS2015-65078-C2 and No. PGC2018-094684-B-C21 (contracts partially funded by FEDER).

\section{APPENDIX A: SAMPLE PREPARATION}

Crystal growth and sample preparation were carried out by the Materials Preparation Center (MPC) of the Ames Laboratory, U.S. Departmetn of Energy. Cu from Luvata Special Products (99.99 wt. \% with respect to specified elements) and distilled Mn from the MPC (99.93 wt. \% with respect to all elements) were arc melted several times under Ar and then drop cast in a water-chilled copper mold. The resulting ingot was placed in a Bridgman-style alumina crucible and heated under vacuum in a resistance Bridgman furnace to $1050^{\circ} \mathrm{C}$, just above the melting point. The chamber was then backfilled to a pressure of 60 psi with high-purity argon to minimize the vaporization of $\mathrm{Mn}$ during the growth. The ingot was then further heated to $1300^{\circ} \mathrm{C}$ and held for $1 \mathrm{~h}$ to ensure complete melting and time for the heat zone to reach a stable state. The ingot was withdrawn from the heat zone at a rate of $3 \mathrm{~mm} / \mathrm{h}$. About $1 / 3$ of the crucible stuck to the alloy. The ingot was finally freed after alternating between hitting with a small punch and hammer and submerging in liquid nitrogen.

Cross sections 1-2 mm thick were taken from near the start of the crystal growth and from the end for characterization. One side of each was polished and looked at optically and with $\mathrm{X}$-ray fluorescence (XRF). From the XRF measurements, the sample was found to be single phase, and the end of the growth was found to be Mn rich. The samples were then etched in a $25 \%$ by volume solution of nitric acid in water. Optically, the start of the growth is a single-phase single crystal, while the end of the growth has large grains with the second phase along the grain boundaries. Small pits were seen both optically and by XRF. The pits could be minimized by 


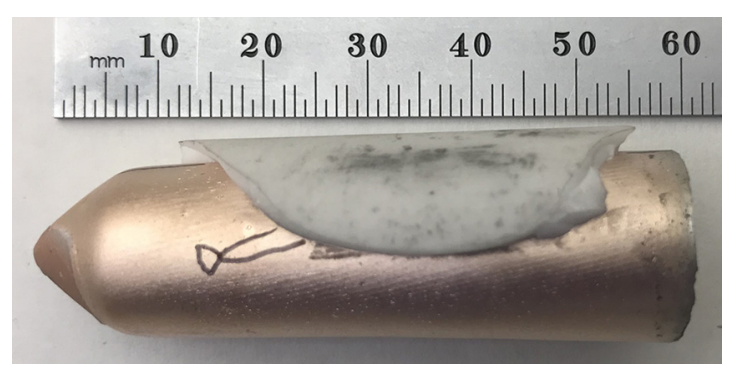

FIG. 5. The as-grown crystal with part of the alumina crucible still attached. A small secondary grain is outlined by a marker. Later, acid etching of the ingot reduced the size and number of the secondary grains, indicating that they are shallow.

various polishing techniques but not gotten rid of. Figure 5 displays the as-grown crystal.

Only the body portion of the crystal growth was used for the experiments. The ends of the growth were looked at as part of the characterization but were not used because the end of the growth contained multiple grains and a second phase. An additional examination of the body was done to ensure that enough of the body had been cut away to remove those unwanted elements. The small, shallow grains that remained on one end of the body were avoided when cutting the sample to be measured. As mentioned above, XRF showed the body of the crystal growth to be single phase. The composition gradient is gradual and smooth, and there was no evidence of a Mn inhomogeneity seen in either the XRF or optical characterization.

Further investigation was done by polishing the cut ends of the ingot body, followed by etching. No evidence of a second phase was seen, and only occasional small, shallow secondary grains were found. In the Bridgman method, it is not unusual for the very end of the growth to be different because of the accumulation of rejected elements and impurities ahead of the growth front. This would account for the change in growth habit (increased number of grains), presence of the second phase, and overall Mn-rich composition seen at the end of the growth but not in the body. Laue x-ray diffraction along the length of the body (Fig. 6) confirms that the majority of the body is one single grain.

\section{APPENDIX B: THE PARAMETERS FOR COMPUTING THE JOSEPHSON LENGTH}

We follow here Ref. [9]. The first step is converting the temperature to Janus units,

$$
T^{(J)}=\frac{T}{T_{\mathrm{c}}} T_{\mathrm{c}}^{(J)}, \quad T_{\mathrm{c}}^{(J)}=1.102 .
$$

Therefore, our working temperature $T=28 \mathrm{~K}$ translates to $T^{(J)}=0.98$.

Next, we need to recall that the only thing we know for sure about this length scale is how it scales:

$\ell_{\mathrm{J}}\left(T^{(J)}\right)=\frac{b_{0}+b_{1}\left(T_{\mathrm{c}}^{(J)}-T^{(J)}\right)^{\nu}+b_{2}\left(T_{\mathrm{c}}^{(J)}-T^{(J)}\right)^{\omega \nu}}{\left(T_{\mathrm{c}}^{(J)}-T^{(J)}\right)^{-v}}$,

where we include analytic $\left(b_{1}\right)$ and confluent $\left(b_{2}\right)$ scaling corrections with $\omega=1.12(10), v=2.56(4)$, and
FIG. 6. The Laue $\mathrm{x}$-ray diffraction pattern of the sample confirms it is a single crystal; the $\mathrm{Cu}_{94} \mathrm{Mn}_{6}$ cube sample was etched in $15 \%$ nitric acid. The 6 at. $\%$ Mn concentration was estimated from scaling using the observed temperature $\left(T_{g}=31.5 \mathrm{~K}\right)$ at which the remanence disappeared.

$T_{\mathrm{c}}^{(J)}=1.102(3)$ [12]. Now, although there is no unique way of fixing the overall scale $b_{0}$ (only the quotients $b_{1} / b_{0}$ and $b_{2} / b_{0}$ can be fixed in a unique way), we shall adhere to the normalization of Ref. [9], so that we can make a comparison to their data in a direct way:

$$
\begin{aligned}
& b_{0}=0.101507196509469, \\
& b_{1}=0.372545152960033, \\
& b_{2}=0.199692833647175 .
\end{aligned}
$$

With this convention for $b_{0}$, at the working temperature $T=0.98$ we have $\ell_{\mathrm{J}}(0.98)=21.82 a_{0}$.

\section{APPENDIX C: THE REPLICON EXPONENT AND THE SELF-CONSISTENT COMPUTATION OF $\xi$}

Let us recall from the main text the relation linking the number of correlated spins $N_{c}$ with the correlation length $\xi$ :

$$
N_{c} \approx\left(\frac{\xi}{a_{0}}\right)^{d_{f}}
$$

where $d_{f}$ is the fractal dimension equal to $d_{f}=d-\theta / 2$ ( $d=3$ is the space dimension, while $\theta$ is the so-called replicon exponent [19]). The quantity directly measured in the experiment is $N_{c}$, and our goal is to convert it into a length by using the fractal dimension.

Now, the problem with Eq. (C1) is that the replicon exponent, and hence $d_{f}$, depends on both the temperature and $\xi$ through the crossover variable (for the reader's convenience, we repeat here the definitions given in the main text):

$$
x=\frac{\ell_{\mathrm{J}}(T)}{\xi\left(t_{\mathrm{w}}, T\right)} .
$$

The data for $\theta(x(\xi, T))$, as well as a discussion of the asymptotic behavior for small $x$, are given in Sec. $\mathrm{C}$ of the Supplemental Material of [9]. Here, we observe only that the numerical data for $\theta(x(\xi, T))$ are well interpolated as (see 


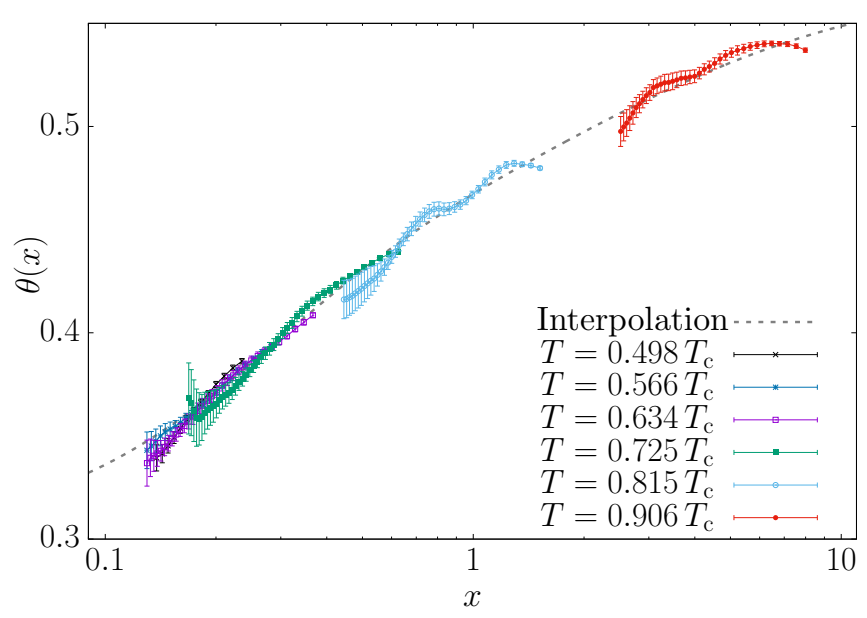

FIG. 7. Data for the replicon exponent, taken from Fig. 5 in the Supplemental Material of Ref. [9], as a function of the crossover variable $x$ defined in Eq. (C2). The black line is the RSB-inspired interpolation in Eq. (C3). The wiggles are due to the extreme data correlation (see, e.g., the discussion of Fig. 1 in Ref. [25]).

Fig. 7)

$$
\theta(x)=\theta_{0}+d_{1}\left(\frac{x}{1+e_{1} x}\right)^{2-\theta_{0}}+d_{2}\left(\frac{x}{1+e_{2} x}\right)^{3-\theta_{0}},
$$

with numerical coefficients

$$
\begin{gathered}
\theta_{0}=0.303980, \\
e_{1}=1.38179 \\
d_{1}=2.72489 \\
e_{2}=2.12634 \\
d_{2}=-9.98359
\end{gathered}
$$

Let us emphasize that the interpolation (C3) is consistent with the replica symmetry breaking (RSB) asymptotic analysis (for small $x$ ) presented in the Supplemental Material of [9]. Yet Eq. (C3) can be applied as well for larger $x$ if needed.

Now, droplet model supporters will object that $\theta_{0}$ should be zero (according to their theory). However, the RSB/droplets controversy is immaterial here: data can be fitted as well to the droplet model (see [9]), but the droplets' fit starts to depart significantly from the RSB interpolation in Eq. (C3) only for $x<0.065$. Because we aim to use the interpolation in the range $x \geqslant 0.0915$, we do not need to worry about the RSB/droplets controversy. After these preliminaries, the selfconsistent computation is straightforward. In order to obtain $\theta$ as a function of the measured number of correlated spins $N_{c}$ (see Fig. 8), we just need to vary $\xi$ parametrically and compute both $\theta\left(x=\ell_{\mathrm{J}}(T=28 \mathrm{~K}) / \xi\right)$ from Eq. (C3) and $N_{c}$ from

$$
N_{c}=\left(\xi / a_{0}\right)^{d_{f}\left[x=\ell_{\mathrm{J}}(28 \mathrm{~K}) / \xi\right]}, \quad d_{f}(x)=3-\frac{\theta(x)}{2} .
$$

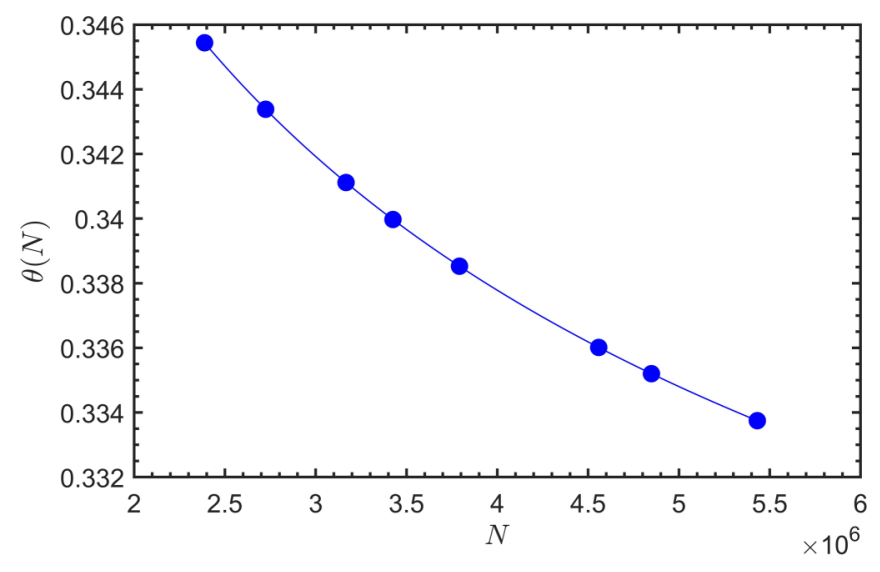

FIG. 8. Self-consistent computation of the replicon exponent $\theta$. By varying $\xi$, we obtain a parametric plot of $\theta=\theta\left[x=\ell_{\mathrm{J}}(T=\right.$ $28 K) / \xi]$, Eq. (C3), as a function of the measured number of correlated spins $N_{c}$ [see Eq. (C9)]. The dots are the appropriate values of $\theta$ for our measured $N_{c}$. Note that $\theta$ is essentially constant in the experimentally relevant range of $N_{c}$.

\section{APPENDIX D: DETAILS OF THE EXTRAPOLATION OF THE AGING RATE}

Our basic quantity will be the (bare) aging rate

$$
z(T, \xi)=\frac{d \ln t_{\mathrm{w}}}{d \ln \xi}
$$

(the renormalized aging rate considered in the main text is just $\left.z_{\mathrm{c}}=z T / T_{\mathrm{c}}\right)$.

Our starting point will be Table III in the Supplemental Material of Ref. [9]. In this table, we find the extrapolated bare aging rates for $\xi_{\text {target }}=38 a_{0}, 76 a_{0}$, and $\infty$, as computed from the convergent ansatz:

$$
z\left(T, \xi_{\text {target }}, \xi_{\min }\right)=z_{\infty}\left(T, \xi_{\min }\right)+\frac{A\left(T, \xi_{\min }\right)}{\xi_{\text {target }}^{\hat{\omega}}} .
$$

In the above expression, $\hat{\omega}=0.35$, and $\xi_{\min }$ is the minimal correlation length considered in their fit. It varies from $\xi_{\min }=3.5 a_{0}$ to $\xi_{\min }=9 a_{0}$ (or less than $9 a_{0}$ at the lowest temperatures).

Our first step was getting the slopes $A\left(T, \xi_{\min }\right)$ from the tabulated values for $\xi_{\text {target }}=38 a_{0}$ and $76 a_{0}$ [instead, $z_{\infty}\left(T, \xi_{\min }\right)$ is directly tabulated]. With this information in our hands, we may compute $z\left(T, \xi_{\text {target }}, \xi_{\min }\right)$ for any value of $\xi_{\text {target }}$ we wish. As for the error estimate, it is only slightly more complicated:

$$
\begin{aligned}
\Delta^{2} z\left(T, \xi_{\text {target }}, \xi_{\min }\right)= & E_{11}^{\left(T, \xi_{\min }\right)}+E_{22}^{\left(T, \xi_{\min }\right)} \frac{1}{\xi_{\text {target }}^{2 \hat{\omega}}} \\
& +E_{12}^{\left(T, \xi_{\min }\right)} \frac{1}{\xi_{\text {target }}^{\hat{\omega}}} .
\end{aligned}
$$

Now, for every $T$ and $\xi_{\min }$, we find error estimates for $\xi_{\text {target }}=\infty, 76 a_{0}$, and $38 a_{0}$ in the table by the Janus Collaboration, which allows us to obtain the constants $E_{11}^{\left(T, \xi_{\min }\right)}$, $E_{22}^{(T, \xi \min )}$. Once we have in our hands the coefficients $E_{22}^{\left(T, \xi_{\min }\right)}$, $E_{11}^{\left(T, \xi_{\min }\right)}$, and $E_{12}^{\left(T, \xi, \xi_{\min }\right)}$, we may compute errors for whatever value of $\xi_{\text {target }}$ we need by using Eq (D3). 
Our next step was obtaining $z\left(T, \xi_{\text {target }}, \xi_{\min }\right)$ for a grid of values $180.26 a_{0} \leqslant \xi_{\text {target }} \leqslant 238.34 a_{0}$. We computed $z\left(T, \xi_{\text {target }}, \xi_{\min }\right)$ for all the values of $\left(T, \xi_{\min }\right)$ in their Table III. We neglected only the few entries where the error for $z\left(T, \xi_{\text {target }}=\infty, \xi_{\min }\right)$ was well above $10 \%$. Then, the estimates for the different $\left(T, \xi_{\min }\right)$ but the same $\xi_{\text {target }}$ were combined as explained in the main text (recall that the renormalized aging rate is $z_{\mathrm{c}}=T z / T_{\mathrm{c}}$ ) by means of a fit to

$$
z_{\mathrm{c}}\left(T, \xi_{\text {target }}, \xi_{\text {min }}\right)=6.69+\frac{\alpha\left(\xi_{\text {target }}\right)}{x_{\min }^{\beta\left(\xi_{\text {target }}\right)}},
$$

where $x_{\min }=\ell_{\mathrm{J}}(T) / \xi_{\min }$. Our final extrapolation was

$$
z_{\mathrm{c}}\left(\xi_{\text {target }}\right)=6.69+\frac{\alpha\left(\xi_{\text {target }}\right)}{x_{\text {target }}^{\beta\left(\xi_{\text {target }}\right)},}, \quad x_{\text {target }}=\frac{\ell_{\mathrm{J}}(28 \mathrm{~K})}{\xi_{\text {target }}} .
$$

The only tricky point needing further discussion regards the computation of errors in $z_{\mathrm{c}}\left(\xi_{\text {target }}\right)$. It is clear that the different data in the fit are extremely correlated (at least those at the same temperature: in Table III of the Supplemental Material of Ref. [9] the Janus Collaboration was simply using the same set of $\xi\left(t_{\mathrm{w}}, T\right)$ and discarding those with $\left.\xi\left(t_{\mathrm{w}}, T\right)<\xi_{\min }\right)$. Under such conditions, the fit's standard errors are not reliable. Hence, in order to estimate errors, we simply repeated the fit for $z_{\mathrm{c}}\left(T, \xi_{\text {target }}, \xi_{\text {min }}\right)$ plus (or minus) the error. In other words, we assumed coherent fluctuations for the whole data set. The errors quoted in the main text are the halved difference between the fit with data plus error and data minus error. A second, far more conservative error estimate would be just to take the error from the data point at the lowest value of $x_{\min }$ included in the fit to Eq. (D4). The conservative error estimate is larger than the error from the halved difference by a factor of 3.75 .
[1] A. Cavagna, Phys. Rep. 476, 51 (2009).

[2] K. Gunnarsson, P. Svedlindh, P. Nordblad, L. Lundgren, H. Aruga, and A. Ito, Phys. Rev. B 43, 8199 (1991).

[3] G. Adam and J. H. Gibbs, J. Chem. Phys. 43, 139 (1965).

[4] Y. G. Joh, R. Orbach, G. G. Wood, J. Hammann, and E. Vincent, Phys. Rev. Lett. 82, 438 (1999).

[5] S. Albert, T. Bauer, M. Michl, G. Biroli, J.-P. Bouchaud, A. Loidl, P. Lunkenheimer, R. Tourbot, C. Wiertel-Gasquet, and F. Ladieu, Science 352, 1308 (2016).

[6] General theoretical arguments suggest that $z_{\mathrm{c}}$ is also $\xi$ independent at exactly $T=T_{\mathrm{c}}$ [26]. Only if $z_{\mathrm{c}}$ is $\xi$ independent do we have a power-law scaling $t_{\mathrm{w}} \propto \xi^{T_{\mathrm{c}} z_{\mathrm{c}} / T}$. If $z_{\mathrm{c}}$ grows with $\xi$, as we find here, we encounter a dynamics slower than a power law (for instance, an activated dynamics with free-energy barriers $\Delta \propto \xi^{\Psi}$ for some $\Psi>0$ ).

[7] Q. Zhai, D. C. Harrison, D. Tennant, E. D. Dahlberg, G. G. Kenning, and R. L. Orbach, Phys. Rev. B 95, 054304 (2017).

[8] G. G. Kenning, D. M. Tennant, C. M. Rost, F. G. da Silva, B. J. Walters, Q. Zhai, D. C. Harrison, E. D. Dahlberg, and R. L. Orbach, Phys. Rev. B 98, 104436 (2018).

[9] M. Baity-Jesi, E. Calore, A. Cruz, L. A. Fernandez, J. M. Gil-Narvion, A. Gordillo-Guerrero, D. Iñiguez, A. Maiorano, E. Marinari, V. Martin-Mayor, J. Moreno-Gordo, A. MuñozSudupe, D. Navarro, G. Parisi, S. Perez-Gaviro, F. RicciTersenghi, J. J. Ruiz-Lorenzo, S. F. Schifano, B. Seoane, A. Tarancon, R. Tripiccione, and D. Yllanes (Janus Collaboration), Phys. Rev. Lett. 120, 267203 (2018).

[10] M. Baity-Jesi, R. A. Baños, A. Cruz, L. A. Fernandez, J. M. Gil-Narvion, A. Gordillo-Guerrero, D. Iniguez, A. Maiorano, F. Mantovani, E. Marinari, V. Martín-Mayor, J. Monforte-Garcia, A. Muñoz Sudupe, D. Navarro, G. Parisi, S. Perez-Gaviro, M. Pivanti, F. Ricci-Tersenghi, J. J. Ruiz-Lorenzo, S. F. Schifano, B. Seoane, A. Tarancon, R. Tripiccione, and D. Yllanes (Janus Collaboration) Comput. Phys. Commun. 185, 550 (2014).

[11] B. D. Josephson, Phys. Lett. 21, 608 (1966).

[12] M. Baity-Jesi, R. A. Banos, A. Cruz, L. A. Fernandez, J. M. Gil-Narvion, A. Gordillo-Guerrero, D. Iñiguez, A. Maiorano, F. Mantovani, E. Marinari, V. Martin-Mayor, J. Monforte-Garcia, A. Muñoz Sudupe, D. Navarro, G. Parisi, S. Perez-Gaviro,
M. Pivanti, F. Ricci-Tersenghi, J. J. Ruiz-Lorenzo, S. F. Schifano, B. Seoane, A. Tarancon, R. Tripiccione, and D. Yllanes (Janus Collaboration), Phys. Rev. B 88, 224416 (2013).

[13] A. J. Bray and M. A. Moore, J. Phys. C 15, 3897 (1982).

[14] M. Baity-Jesi, L. A. Fernandez, V. Martín-Mayor, and J. M. Sanz, Phys. Rev. B 89, 014202 (2014).

[15] D. J. Amit and V. Martín-Mayor, Field Theory, the Renormalization Group and Critical Phenomena, 3rd ed. (World Scientific, Singapore, 2005).

[16] S. Guchhait and R. L. Orbach, Phys. Rev. Lett. 118, 157203 (2017).

[17] E. Wandersman, V. Dupuis, E. Dubois, R. Perzynski, S. Nakamae, and E. Vincent, Europhys. Lett. 84, 37011 (2008).

[18] S. Nair and A. K. Nigam, Phys. Rev. B 75, 214415 (2007).

[19] M. Baity-Jesi, E. Calore, A. Cruz, L. A. Fernandez, J. M. Gil-Narvion, A. Gordillo-Guerrero, D. Iñiguez, A. Maiorano, E. Marinari, V. Martin-Mayor, J. Monforte-Garcia, A. MuñozSudupe, D. Navarro, G. Parisi, S. Perez-Gaviro, F. RicciTersenghi, J. J. Ruiz-Lorenzo, S. F. Schifano, B. Seoane, A. Tarancon, R. Tripiccione, and D. Yllanes (Janus Collaboration) Phys. Rev. Lett. 118, 157202 (2017).

[20] The reader will note that the right-hand side of Eq. (5) could be modified by a prefactor of order 1 . This is why we are using an approximate sign in the equation, rather than an equals sign. However, the comparison with the simulations turns out to be satisfactory by assuming that the prefactor is exactly 1 . It is very possible that carrying out our program in future experiments with increased accuracy will require a more precise determination of this prefactor.

[21] Amusingly, although the droplets model [27-29] and the replica symmetry breaking (RSB) theory [30] differ in their expectation for $\theta(\xi \rightarrow \infty)$ [the droplet prediction is $\theta(\xi \rightarrow \infty)=0$ and $d=d_{\mathrm{f}}$, while RSB predicts $\theta(\xi \rightarrow \infty)>0$ and $\left.d_{\mathrm{f}}<d\right]$, the two theories quantitatively agree in their predicted behavior for $\theta(\xi)$ in our range of $\xi[9]$.

[22] J.-P. Bouchaud, V. Dupuis, J. Hammann, and E. Vincent, Phys. Rev. B 65, 024439 (2001). 
[23] L. Berthier and J.-P. Bouchaud, Phys. Rev. B 66, 054404 (2002).

[24] Note that Eq. (7) correctly predicts $z_{\mathrm{c}}\left(T_{\mathrm{c}}\right)=6.69$ because $x=\infty$ at $T_{\mathrm{c}}$ for all $\xi$.

[25] F. Belletti, M. Cotallo, A. Cruz, L. A. Fernandez, A. GordilloGuerrero, M. Guidetti, A. Maiorano, F. Mantovani, E. Marinari, V. Martin-Mayor, A. Muñoz Sudupe, D. Navarro, G. Parisi, S. Perez-Gaviro, J. J. Ruiz-Lorenzo, S. F. Schifano, D. Sciretti, A. Tarancon, R. Tripiccione, J. L. Velasco, and D. Yllanes Phys. Rev. Lett. 101, 157201 (2008).
[26] J. Zinn-Justin, Quantum Field Theory and Critical Phenomena, 4th ed. (Clarendon, Oxford, 2005).

[27] W. L. McMillan, Phys. Rev. B 28, 5216 (1983).

[28] A. J. Bray and M. A. Moore, in Heidelberg Colloquium on Glassy Dynamics, edited by J. L. van Hemmen and I. Morgenstern, Lecture Notes in Physics Vol. 275 (Springer, Berlin, 1987).

[29] D. S. Fisher and D. A. Huse, Phys. Rev. Lett. 56, 1601 (1986).

[30] E. Marinari, G. Parisi, F. Ricci-Tersenghi, J. J. Ruiz-Lorenzo, and F. Zuliani, J. Stat. Phys. 98, 973 (2000). 